\title{
Irinotecan/Panitumumab/Vemurafenib Regimen
}

National Cancer Institute

\section{Source}

National Cancer Institute. Irinotecan/Panitumumab/Vemurafenib Regimen. NCI

Thesaurus. Code C153482.

A chemoimmunotherapy regimen consisting of irinotecan, panitumumab and vemurafenib that can be used for the treatment of colon cancer. 